\section{Gut Microbiome and Liver Diseases}

independent cohort. Thus microbiota-targeted biomarkers may be a powerful tool for diagnosis of different diseases.

\section{COMMENTS}

The human gut microbiome (also called gut flora) consists of a complex combination of microbes of different typesthese are mainly bacteria; and, in addition, some viruses, fungi and protozoa. The total population of these bacterial cells outnumber the human cells by 10 folds. The composition and the density of the organisms vary across the gastrointestinal tract, the largest numbers are found in the large intestine. The predominant microbiota seen in the human gut belongs to the following four phyla: Firmicutes, Bacteroidetes, Proteobacteria and Actinobacteria. ${ }^{1}$

Gut microbiota play an important role in the development of immune system and contribute to a variety of metabolic processes. The species composition varies and is determined by environment and diet. They have been implicated in the pathogenesis of non-alcoholic liver disease, development of complications in cirrhosis (hepatic encephalopathy, spontaneous bacterial peritonitis and esophageal variceal bleeding). A recent study documented a change in the ratio of autochthonous (indigenous) to non-autochthonous microbiota-cirrhosis dysbiosis ratio (CDR) - with the development of decompensation. The CDR was highest in controls (2.05) followed by compensated (0.89) and decompensated $(0.66)$ patients $(P<$ 0.0001 ) and negatively correlated with endotoxin. ${ }^{2}$

Thus it is important to understand the profiles of these microbes in order to develop strategies to modify disease outcomes. In a phase I trial, Lactobacillus GG has been shown to be associated with a reduction in endotoxemia and dysbiosis. The future trials will focus on the modification of the gut microbiome, in order to improve the prognosis of disease.

The various techniques used for detection of intestinal microbiota include culture based and culture independent techniques (16S rRNA gene based approach: species identification, metagenomics: sequencing of entire bacterial genome, metatranscriptomics: messenger RNAs, metaproteomics: proteins, metabolomics: metabolites). ${ }^{3}$

In this study by Qin et al from China, the authors in first step constructed a gene catalogue from 98 Chinese patients with cirrhosis and 83 healthy controls using the Illumina-based metagenomic sequencing. ${ }^{4}$ This catalogue was then compared with other three available gut microbial catalogues: MetaHIT (European), ${ }^{4}$ US National Institutes of Health Human Microbiome Project (HMP) ${ }^{5}$ and Type 2 diabetes. ${ }^{6}$

Bacteroidetes and Firmicutes were the most common microbes in both cirrhosis and healthy controls. There were differences in the proportion of various microbes between the two groups at the phylum level-patients with cirrhosis had fewer Bacteroidetes, whereas the proportions of
Received: 28.8.2014; Accepted: 28.8.2014; Available online: 18.9.2014

Abbreviations: CDR: cirrhosis dysbiosis ratio; HMP: Human Microbiome Project

http://dx.doi.org/10.1016/j.jceh.2014.08.010 
Proteobacteria and Fusobacteria were more in these patients as compared to controls. Among cirrhosis patients, higher proportion of Veillonella, Streptococcus, Clostridium and Prevotella and lower proportions of Bacteroides were seen. The authors raise the possibility of the role of altered microbiota in the causation of cirrhosis. In the study, the authors have not provided a subgroup analysis of differences in the microbiota in different etiologies of cirrhosis, without which such a conclusion is a bit premature. There is a need to further explore the role of gut microbiota in disease progression in different etiologies of cirrhosis (viral, alcohol, non-alcoholic fatty liver disease and autoimmune).

In addition, the metagenomic species (i.e. clustering of microbial genes according to their abundance) were different between the cirrhosis and control groups. There were similarities between the metagenomic species seen in the Chinese and Danish cohort across healthy individuals suggesting similar microbial profile across different population groups. Among the abundant species seen in cirrhosis patients, around 50\% were suggestive of oral isolate, raising the possibility of oral commensals invading the gut. Their role in the pathogenesis and development of decompensation needs to be evaluated.

The authors selected 15 microbial genes as biomarkers to create a patient discrimination index to differentiate patients with cirrhosis from controls, and these results were validated on another cohort. They concluded that these specific genes could accurately distinguish patients with cirrhosis from healthy individuals. The results of the study have opened a new window of therapeutic targets - either use of probiotics (containing 'good' bacteria) or specific targets (to remove the 'bad' bacteria) and tilt the balance towards the side of favourable bacteria. Preliminary studies have shown that probiotics can be used to alter the gut microbiome with good effects. With time we will come to know how well this translates into clinical benefit for the human population.

\section{CONFLICTS OF INTEREST}

The author have none to declare.

\section{REFERENCES}

1. Eckburg PB, Bik EM, Bernstein CN, et al. Diversity of the human intestinal microbial flora. Science. 2005;308:1635-1638.

2. Bajaj JS, Heuman DM, Hylemon PB, et al. Altered profile of human gut microbiome is associated with cirrhosis and its complications. J Hepatol. 2014;60:940-947.

3. Simrén M, Barbara G, Flint HJ, et al, Rome Foundation Committee. Intestinal microbiota in functional bowel disorders: a Rome foundation report. Gut. 2013;62:159-176.

4. Qin J, Li R, Raes J, et al, MetaHIT Consortium. A human gut microbial gene catalogue established by metagenomic sequencing. Nature. 2010;464:59-65.

5. Human Microbiome Project Consortium. A framework for human microbiome research. Nature. 2012;486:215-221.

6. Qin J, Li Y, Cai Z, et al. A metagenome-wide association study of gut microbiota in type 2 diabetes. Nature. 2012;490(7418): 55-60.

$$
\begin{array}{r}
\text { Shalimar } \\
\text { Department of Gastroenterology, } \\
\text { All India Institute of Medical Sciences, New Delhi, India }
\end{array}
$$

Tel.: +919968405815

E-mail: drshalimar@yahoo.com 